\title{
Colloidal Synthesis of Bipolar Off-Stoichiometric Gallium Iron Oxide Spinel-Type Nanocrystals with Near-IR Plasmon Resonance
}

\author{
Carmine Urso, ${ }^{\dagger, \ddagger}$ Mariam Barawi, ${ }^{\S}$ Roberto Gaspari, ${ }^{\dagger}, \|$ Gianluca Sirigu, ${ }^{\perp}$ Ilka Kriegel, ${ }^{\dagger}$
} Margherita Zavelani-Rossi, ${ }^{\#, \nabla}$ ๑ Francesco Scotognella, ${ }^{\perp, \nabla}$ Michele Manca, ${ }^{\S}$ Mirko Prato, ${ }^{\dagger}$ Luca De Trizio, ${ }^{*}{ }^{\dagger}$ a and Liberato Manna* ${ }^{* \dagger}$

${ }^{\dagger}$ Nanochemistry Department, Istituto Italiano di Tecnologia (IIT), via Morego 30, Genova, Italy

${ }^{\ddagger}$ Department of Chemistry and Industrial Chemistry, University of Genoa, via Dodecaneso 31, Genova, Italy

${ }^{\S}$ Center for Biomolecular Nanotechnologies, Istituto Italiano di Tecnologia (IIT), Via Barsanti 14, 73010 Arnesano (Lecce), Italy

"CompuNet, Istituto Italiano di Tecnologia (IIT), via Morego, 30, 16163 Genova, Italy

${ }^{\perp}$ Dipartimento di Fisica, Politecnico di Milano, P.za Leonardo da Vinci 32, 20133 Milano, Italy

\#Dipartimento di Energia, Politecnico di Milano, via Ponzio 34/3, 20133 Milano, Italy

${ }^{\nabla}$ Istituto di Fotonica e Nanotecnologie CNR, Piazza Leonardo da Vinci 32, 20133 Milano, Italy

Supporting Information

ABSTRACT: We report the colloidal synthesis of $\sim 5.5 \mathrm{~nm}$ inverse spinel-type oxide $\mathrm{Ga}_{2} \mathrm{FeO}_{4}$ (GFO) nanocrystals (NCs) with control over the gallium and iron content. As recently theoretically predicted, some classes of spinel-type oxide materials can be intrinsically doped by means of structural disorder and/or change in stoichiometry. Here we show that, indeed, while stoichiometric $\mathrm{Ga}_{2} \mathrm{FeO}_{4} \mathrm{NCs}$ are intrinsic small bandgap semiconductors, off-stoichiometric GFO NCs, produced under either Fe-rich or Ga-rich conditions, behave as degenerately doped semiconductors. As a consequence of the generation of free carriers, both Fe-rich and Ga-rich GFO

NCs exhibit a localized surface plasmon resonance in the near-infrared at $\sim 1000 \mathrm{~nm}$, as confirmed by our pump-probe absorption measurements. Noteworthy, the photoelectrochemical characterization of our GFO NCs reveal that the majority carriers are holes in Fe-rich samples, and electrons in Ga-rich ones, highlighting the bipolar nature of this material. The behavior of such off-stoichiometric NCs was explained by our density functional theory calculations as follows: the substitution of $\mathrm{Ga}^{3+}$ by $\mathrm{Fe}^{2+}$ ions, occurring in Fe-rich conditions, can generate free holes (p-type doping), while the replacement of $\mathrm{Fe}^{2+}$ by Ga $^{3+}$ cations, taking place in Ga-rich samples, produces free electrons (n-type doping). These findings underscore the potential relevance of spinel-type oxides as p-type transparent conductive oxides and as plasmonic semiconductors.

\section{INTRODUCTION}

In the past few years, colloidal nanocrystals (NCs) of metal oxides have generated much interest for tunable plasmonics as their electrical conductivity and their localized surface plasmon resonance (LSPR) can be controlled by means of doping. ${ }^{1-11}$ Unlike conventional metallic plasmonic NCs (e.g., Au and Ag) that have a fixed free-electron concentration, semiconductor NCs, such as metal oxides, copper chalcogenides, copper pnictides and silicon, are unique as their carrier density, and thus, the absorption features arising from the LSPR, can be actively modulated across visible, near-infrared (NIR), and midIR wavelengths by varying the density of dopants. ${ }^{2,7,8,12-16}$ Also, it has been shown that the surface plasmon resonance of such NCs can be dynamically and reversibly tuned by postsynthetic electrochemical modulation of the carrier concentration. ${ }^{17}$ Thanks to these properties, doped metal oxide NCs are being harnessed for an increasing number of applications. ${ }^{6,8,17-26}$

The generation of free charge carriers in metal oxide NCs can occur by intrinsic doping (i.e., by lattice vacancies or interstitials), by extrinsic aliovalent substitutional doping, and, less commonly, by extrinsic interstitial doping. ${ }^{27,28} \mathrm{WO}_{3-x}$ and $\mathrm{MoO}_{3-x} \mathrm{NCs}$, for example, exhibit a LSPR due to the presence of free electrons originated by oxygen vacancies. ${ }^{29,30}$ As the control over the concentration of intrinsic defects is generally not trivial in metal oxide NCs, substitutional doping with aliovalent cations has been more widely exploited. A fine modulation of the doping level, hence of the plasmon resonance, has been demonstrated for many n-type systems such as $\mathrm{Sn}$-doped $\mathrm{In}_{2} \mathrm{O}_{3}$ (ITO), $\mathrm{M}^{3+}$-doped $\mathrm{ZnO}(\mathrm{M}=\mathrm{Al}$, Ga,

Received: October 24, 2016

Published: December 22, 2016 
or In), Sb-doped $\mathrm{SnO}_{2}$, In-doped $\mathrm{CdO}, \mathrm{Nb}$-doped $\mathrm{TiO}_{2}$ and, more recently, Ce-doped $\mathrm{In}_{2} \mathrm{O}_{3} \mathrm{NCs} .^{31-40}$ Interstitial doping of transition metal oxide NCs to generate free electrons, as in the case of $\mathrm{Cs}_{x} \mathrm{WO}_{3} \mathrm{NCs}$, and the cation-anion codoping of $\mathrm{CdO}$ NCs (with $\mathrm{In}^{3+}$ or $\mathrm{Sn}^{4+}$ and $\mathrm{F}^{-}$ions) have also been reported. ${ }^{41-43}$

The success of such n-type metal oxides in semiconductor technologies (e.g., in thin-film transistors) has then raised interest in p-type oxides (with particular interest in the production of all-oxide $\mathrm{p}-\mathrm{n}$ junctions), whose performance, to date, has not yet reached that of the n-type ones. ${ }^{25,26,44-48}$ Indeed, the ionicity of the aforementioned metal oxides, whose valence band maximum (VBM) is dominated by $\mathrm{O}^{2-} 2 \mathrm{p}$ states, allows little or no p-type doping as generated deep lying holes are strongly localized on oxygen sites. Moreover, the formation of acceptor defects in such compounds is always compensated by the formation of stable oxygen vacancies (which have low formation energies) under equilibrium conditions. ${ }^{2,25,44,45,47-49}$ Effective p-type doping is believed to be achieved only in oxide materials characterized by more covalent metal-oxygen bonds, which should lead to an upward bowing of the VBM and, at the same time, to an extended valence-band structure that delocalizes the positive holes. ${ }^{27,45,50}$ Examples are $\mathrm{NiO}$, $\mathrm{Bi}_{2} \mathrm{O}_{3}, \mathrm{SnO}$, and $\mathrm{Cu}^{\mathrm{I}}$-based oxides, such as $\mathrm{Cu}_{2} \mathrm{O}, \mathrm{CuMO}_{2}$ $(\mathrm{M}=\mathrm{Al}, \mathrm{Ga}$, In, $\mathrm{Cr}$, etc.) with the delafossite structure and $\mathrm{SrCu}_{2} \mathrm{O}_{2}$. $25,27,44,48,49,51-55$ Unfortunately all these materials suffer from low hole concentration and/or low carrier mobility, despite considerable efforts having been made to improve their electrical properties. ${ }^{4,54}$

An emerging class of oxide materials, that has shown great potentialities for both $\mathrm{n}$ and, especially, p-type doping, is represented by spinel oxides. They have a general $\mathrm{A}_{2} \mathrm{BO}_{4}$ chemical formula, with $\mathrm{O}^{2-}$ anions forming a cubic closepacked framework in which $\mathrm{A}^{3+}$ and $\mathrm{B}^{2+}$ cations occupy two inequivalent lattice sites: one with four tetrahedrally $\left(T_{d}\right)$ coordinated nearest neighbor oxygen atoms and one with six octahedrally $\left(\mathrm{O}_{\mathrm{h}}\right)$ coordinated oxygens. At low temperature the location of the cations is fixed: for example, in ordered-normal spinels $\mathrm{A}^{3+}$ cations occupy $\mathrm{O}_{h}$ sites whereas the $\mathrm{B}^{2+}$ cations occupy $\mathrm{T}_{d}$ sites, resulting in a closed-shell insulator. At finite temperature two different channels of disorder typically occur: (1) the formation of antisite defects arising from a crosssubstitution of the A and B cations (e.g., cations that usually occupy $\mathrm{T}_{\mathrm{d}}$ sites reside, instead, on $\mathrm{O}_{\mathrm{h}}$ sites or vice versa), while the overall stoichiometry is preserved (i.e., A/B/O ratio of $2: 1: 4) ;(2)$ a change of stoichiometry, whereby the $\mathrm{A} / \mathrm{B} / \mathrm{O}$ ratio is altered from $2: 1: 4$. One intriguing aspect of spinel oxides is that a fine-tuning of the stoichiometry and the concentration of antisite defects can, in principle, lead to a control over the doping type and the free carrier density of these materials. ${ }^{56}$ This was theoretically predicted by Paudel et al., ${ }^{57}$ and, indeed, both antisite defects and off-stoichiometry have been shown to represent the two main sources of electrical conductivity in these compounds. Notably, the formation energy of antisite defects is much lower than that of vacancies and interstitials, such that the latter two do not contribute to the level of doping of spinel oxides. ${ }^{57}$ To date, few reports have demonstrated the possibility to prepare p-type films of $\mathrm{A}_{2} \mathrm{ZnO}_{4}$ $\left(\mathrm{A}=\mathrm{Co}^{3+}, \mathrm{Rh}^{3+}\right.$, and $\left.\mathrm{Ir}^{3+}\right)$ spinel oxides. ${ }^{58,59}$ Indeed, as predicted by calculations, Perkins et al. have shown that an excess of the lower-valent cation $\left(\mathrm{B}^{2+}\right)$ in $\mathrm{Co}_{2} \mathrm{ZnO}_{4}$ enhances its p-type behavior. ${ }^{60}$
Motivated by these works, we have selected a spinel oxide material in which both $\mathrm{p}$ and n-type doping can be induced, in principle, by off-stoichiometry: $\mathrm{Ga}_{2} \mathrm{FeO}_{4}$ (GFO). ${ }^{57}$ The interesting feature of GFO, as shown by a recent work of Burnett et al., ${ }^{61}$ is that the material can sustain a certain range of stoichiometries while retaining its spinel structure. Also, as predicted by Paudel et al., 57 disorder in this material can produce either donor levels close to the conduction band minimum (CBM, n-type doping) or acceptor states close to the VBM (p-type doping). Here, we report a colloidal synthesis of GFO NCs with control over the composition of the resulting particles. By varying the relative amount of gallium and iron precursors, it was possible to synthesize both stoichiometric and off-stoichiometric (i.e., Ga-rich or Fe-rich) GFO NCs. Interestingly, as corroborated by our transient photovoltage measurements, off-stoichiometric GFO NCs behaved as degenerately doped semiconductors, with the character varying from n-type to p-type going from Ga-rich to Fe-rich conditions (bipolar nature). As a consequence of the doping, offstoichiometric samples exhibited a LSPR in the NIR, as confirmed by our pump-probe experiments, making GFO NCs a good candidate for plasmonic applications.

\section{EXPERIMENTAL SECTION}

Chemicals. Oleylamine (Olam, 70\%), oleic acid (OAc, 90\%), 1octadecene (ODE, 90\%), and gallium(III) acetylacetonate $\left(\mathrm{Ga}(\mathrm{acac})_{3}\right.$, 99.99\%) were purchased from Sigma-Aldrich. Ferrous acetylacetonate $\left(\mathrm{Fe}(\mathrm{acac})_{2}\right)$ was purchased from Molekula. Ethanol (ACS grade, $>99.8 \%$ ), chloroform (ACS grade, $>99.8 \%$ ), toluene (ACS grade, $>99.7 \%$ ), tetrahydrofuran (THF, CHROMA SOLV Plus, $>99.9 \%$ ), and tetrachloroethylene (TCE, ACS grade, $\geq 99.0 \%$ ) were purchased from Sigma-Aldrich. All chemicals were used without further purification.

Synthesis of $\mathrm{Ga}_{2} \mathrm{FeO}_{4}$ nanocrystals. In a typical synthesis of stoichiometric $\mathrm{Ga}_{2} \mathrm{FeO}_{4} \mathrm{NCs}, 250 \mathrm{mg}(0.68 \mathrm{mmol})$ of $\mathrm{Ga}(\mathrm{acac})_{3}$ and $89 \mathrm{mg}(0.35 \mathrm{mmol})$ of $\mathrm{Fe}(\mathrm{acac})_{2}$ were dissolved in a mixture of 2.15 $\mathrm{mL}(10 \mathrm{mmol})$ of Olam, $1.25 \mathrm{~mL}(5 \mathrm{mmol})$ of OAc, and $3 \mathrm{~mL}$ of ODE in a three-neck flask at $80{ }^{\circ} \mathrm{C}$. The solution was then degassed under vacuum at $130{ }^{\circ} \mathrm{C}$ for $1 \mathrm{~h}$ and then heated to $300{ }^{\circ} \mathrm{C}$ under nitrogen flux for $1 \mathrm{~h}$. The reaction mixture was then cooled to room temperature. The resulting NCs were washed three times by dispersion in chloroform followed by precipitation by addition of ethanol. The brown precipitate was redissolved in chloroform and stored under air. Off-stoichiometric $\mathrm{Ga}_{2} \mathrm{FeO}_{4} \mathrm{NCs}$ were synthesized by varying the $\mathrm{Ga} / \mathrm{Fe}$ precursors molar ratio between 0.7 and 3.3. This was achieved in practice by maintaining fixed the amount of gallium precursor and by adjusting the amount of iron precursor to reach the desired $\mathrm{Ga} / \mathrm{Fe}$ precursor molar ratio.

Transmission Electron Microscopy (TEM) Measurements. The samples were prepared by dropping dilute solutions of NCs onto carbon coated copper grids. Low-resolution TEM measurements were carried out on a JEOL-1100 transmission electron microscope operating at an acceleration voltage of $100 \mathrm{kV}$.

X-ray Diffraction (XRD) Measurements. The XRD analysis was performed on a PANanalytical Empyrean X-ray diffractometer equipped with a $1.8 \mathrm{~kW} \mathrm{Cu} \mathrm{K \alpha}$ ceramic X-ray tube, PIXcel $^{3 \mathrm{D}} 2 \times 2$ area detector and operating at $45 \mathrm{kV}$ and $40 \mathrm{~mA}$. Specimens for the XRD measurements were prepared by dropping a concentrated NCs solution onto a quartz zero-diffraction single crystal substrate. The diffraction patterns were collected at ambient conditions using a parallel beam geometry and symmetric reflection mode. XRD data analysis was carried out using the HighScore 4.1 software from PANalytical.

Elemental Analysis. This was carried out via inductively coupled plasma optical emission spectroscopy (ICP-OES), using a iCAP 6500 Thermo spectrometer. All chemical analyses performed by ICP-OES were affected by a systematic error of about $5 \%$. Samples were dissolved in $\mathrm{HCl} / \mathrm{HNO}_{3}$ 3:1 (v/v). 
UV-Vis-NIR Absorption Spectroscopy. The UV-vis-NIR absorption spectra of the NCs solutions in TCE were recorded using a Varian Cary 5000 UV-vis-NIR absorption spectrophotometer.

Pump-Probe Absorption Spectroscopy. For ultrafast pumpprobe measurements, the laser system employed was based on a Ti:sapphire chirp pulse amplified laser source (Coherent Libra), with a maximum output energy of about $1 \mathrm{~mJ}, 1 \mathrm{kHz}$ repetition rate, a central wavelength of $800 \mathrm{~nm}(1.59 \mathrm{eV})$ and a pulse duration of about $100 \mathrm{fs}$. Pump pulses, at $800 \mathrm{~nm}$, were focused in a spot area of $(400 \times 360)$ $\mu \mathrm{m}^{2}$. Probing was achieved in the near IR region $(870-1500 \mathrm{~nm})$ by using a white light supercontinuum generated in a $3 \mathrm{~mm}$ thick sapphire plate. Chirp-free transient transmission spectra were collected by using a fast optical multichannel analyzer (OMA) with a dechirping algorithm. The spectrometer employed was the InGaAs Bayspec SuperGamut NIR Spectrometer that is able to detect from 870 to 1650 $\mathrm{nm}$. The measured quantity is the normalized transmission change, $\Delta \mathrm{T} / \mathrm{T}$. All measurements were performed at room temperature on sealed samples prepared under nitrogen atmosphere.

X-ray Photoelectron Spectroscopy (XPS). Measurements were performed on a Kratos Axix Ultra DLD spectrometer, using a monochromatic $\mathrm{Al} \mathrm{K} \alpha$ source $(15 \mathrm{kV}, 20 \mathrm{~mA})$. High resolution narrow scans were performed at constant pass energy of $10 \mathrm{eV}$ and steps of $0.10 \mathrm{eV}$. The photoelectrons were detected at takeoff angle of $\phi=0^{\circ}$ with respect to the surface normal. The pressure in the analysis chamber was maintained below $7 \times 10^{-9}$ Torr for data acquisition. The data was converted to VAMAS format and processing using CasaXPS software, version 2.3.16. The binding energy (BE) scale was internally referenced to $\mathrm{C} 1 \mathrm{~s}$ peak $(\mathrm{BE}$ for $\mathrm{C}-\mathrm{C}=284.8 \mathrm{eV}$ ).

Photoelectrochemical Characterization. Pristine colloidal GFO NCs were turned into printable viscous pastes upon the addition of a high boiling solvent (terpineol) and a thickening agent (ethylcellulose) and then employed to prepare crack-free mesoporous films by doctorblade deposition on a silicon substrate and thermal annealing at 390 ${ }^{\circ} \mathrm{C}$ for $15 \mathrm{~min}$. The resulting mesoporous electrodes had an average thickness of $\sim 500 \mathrm{~nm}$ and an active area of $1 \mathrm{~cm}^{2}$. XRD and SEM characterizations were performed in order to ensure that neither sintering nor phase transition of GFO NCs occurred upon the annealing step (see Figure S7 and the Supporting Information (SI) for further details). They were employed as working electrodes in a series of photoelectrochemical measurements carried out in a $0.5 \mathrm{M} \mathrm{Na}_{2} \mathrm{SO}_{3}$ aqueous solution within a three-electrode setup. A platinum foil was used as a counter electrode and an $\mathrm{Ag} / \mathrm{AgCl}$ electrode as a reference. $\mathrm{A}$ withe LED was used as the illumination source. Current/voltage signals were measured through a Autolab PGSTAT302N potentiostat.

The same instrument, which is provided with a FRA2 integrated impedance module, was used to carry out a set of electrochemical impedance spectroscopy (EIS) measurements. In this case the superimposed AC signal was a $10 \mathrm{mV}$ wave with a frequency of 400 $\mathrm{Hz}$. The capacitance of the space charge layer $C_{\mathrm{SC}}$ at the semiconductor/electrolyte interface has been calculated by assuming: ${ }^{62,63}$

$$
1 /\left(w Z_{\text {im }}\right)=C_{\mathrm{SC}}
$$

where $w$ is the angular frequency and $Z_{\text {im }}$ is the imaginary part of the complex impedance. The dependence of $C_{\mathrm{SC}}$ on bias potential is described by the Mott-Schottky equation: ${ }^{64}$

$$
\frac{1}{C_{\mathrm{SC}}{ }^{2}}=\left(\frac{2}{\varepsilon_{\mathrm{SC}} \varepsilon_{0} \mathrm{~N}_{\mathrm{D} / \mathrm{A}} e_{0} A}\right)\left(\left[V-V_{\mathrm{fb}}\right]-\frac{k_{\mathrm{B}} T}{e_{0}}\right)
$$

where $C_{\mathrm{SC}}$ is the measured differential capacitance per area unit, $e_{0}$ is the elementary charge, $\varepsilon_{\mathrm{SC}}$ is the dielectric constant, $\varepsilon_{0}$ is the electrical permittivity of vacuum, $N_{\mathrm{D} / \mathrm{A}}$ is the donor/acceptor density, $V$ is the applied bias potential in volts, $k_{\mathrm{B}}$ is the Boltzmann's constant, $T$ is the temperature $(298 \mathrm{~K}), V_{\mathrm{fb}}$ is the flat band potential, and $A$ is the surface area of the electrode $\left(1 \mathrm{~cm}^{2}\right)$.

Computational Modeling. Density functional theory (DFT) calculations were performed on bulk models of inverse-spinel $\mathrm{Ga}_{2} \mathrm{FeO}_{4}$. Systems corresponding to $2 \times 2 \times 2$ primitive cubic unit cells (i.e., containing $16 \mathrm{Ga}$ and $8 \mathrm{Fe}$ ions) have been considered for the calculations. We used the Perdew-Burke-Enzerhof ${ }^{65}$ functional, norm-conserving pseudopotentials for all elements and the pwscf code. $^{66}$ Respectively, 100 and 400 Ryd were used as cutoff for the plane waves and charge density. A uniform $3 \times 3 \times 3$ mesh was used for Brillouin zone sampling. A Hubbard ${ }^{67}$ correction $U=5 \mathrm{eV}$ has been added on the d-orbitals of Fe. The experimental geometry of the system was employed for the wave function optimization, and the lattice parameter of the cubic cell was kept at the value of $8.36 \AA$. In the starting configuration, all the $\mathrm{T}_{\mathrm{d}}$ sites were occupied by Ga atoms, while $\mathrm{O}_{\mathrm{h}}$ sites had $50 \% \mathrm{Fe}$ and $50 \% \mathrm{Ga}$ occupancy. The actual distribution of $\mathrm{Ga}$ and $\mathrm{Fe}$ atoms is shown in Table S1 of the SI. Starting from this stoichiometric structure, two additional structural models, featuring $\mathrm{Ga}_{\mathrm{Fe}^{2+}}^{3+}$ and $\mathrm{Fe}_{\mathrm{Ga}^{3+}}^{2+}$ substitutions, were derived, as shown in Table S1 of the SI. The wave function optimization for these two models was carried out using the same procedure employed for the stoichiometric structure. The frequency-dependent dielectric function was computed with the random-phase approximation, by employing the epsilon.x tool of pwscf. Interband and intraband smearings of respectively 300 and $100 \mathrm{meV}$ were applied.

\section{RESULTS AND DISCUSSION}

We have developed a colloidal heat-up approach to $\mathrm{Ga}_{2} \mathrm{FeO}_{4}$ NCs in which gallium(III) and iron(II) acetylacetonates were used as metal cation precursors at $300{ }^{\circ} \mathrm{C}$ in the presence of oleic acid and oleylamine. As shown in the TEM micrograph of Figure 1a, the resulting NCs have an average diameter of $5.5 \pm$ $0.8 \mathrm{~nm}$, and an irregular morphology. The XRD analysis of the as-prepared $\mathrm{NCs}$ confirmed their inverse-spinel $\mathrm{Ga}_{2} \mathrm{FeO}_{4}$ structure, with lattice constant $a=8.28 \pm 0.02 \AA$, slightly smaller than that of bulk $\mathrm{Ga}_{2} \mathrm{FeO}_{4}(a=8.36 \AA$, ICSD number 28285) (see Figure $1 \mathrm{~b}$ ). A Ga/Fe ratio of 1.9 was measured by

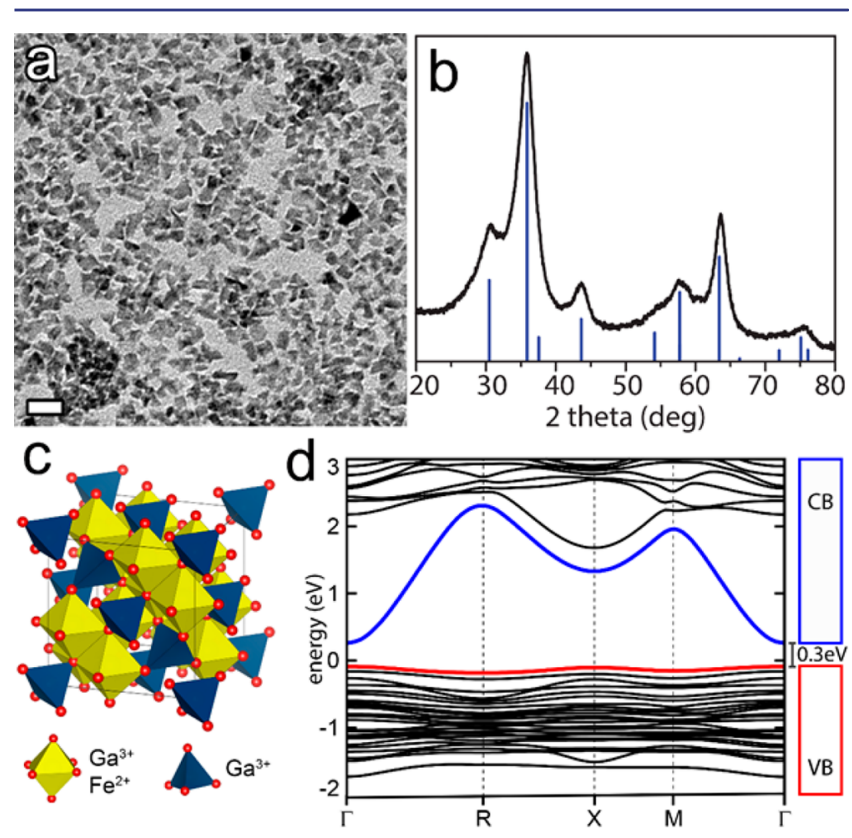

Figure 1. (a) Low resolution TEM image of stoichiometric GFO NCs. Scale bar is $20 \mathrm{~nm}$. (b) XRD pattern from drop-cast solutions of stoichiometric GFO NCs together with the reflections of a $\mathrm{Ga}_{2} \mathrm{FeO}_{4}$ structure having lattice parameters $8.28 \AA$ (calculated starting from the ICSD card No 28285). (c) Schematic representation of the inversespinel $\mathrm{Ga}_{2} \mathrm{FeO}_{4}$-like structure, evidencing the $\mathrm{T}_{\mathrm{d}}$ and $\mathrm{O}_{\mathrm{h}}$ sites occupied by $\mathrm{Ga}^{3+}$ and $\mathrm{Fe}^{2+}$ ions. (d) DFT band structure calculations along the $\Gamma$-R-X-M- $\Gamma$ cubic symmetry line of the $\mathrm{Ga}_{2} \mathrm{FeO}_{4}$ stoichiometric system. The highest occupied band and lowest unoccupied band are colored in red and blue, respectively. A direct band gap of $0.3 \mathrm{eV}$ is visible at $\Gamma$. 

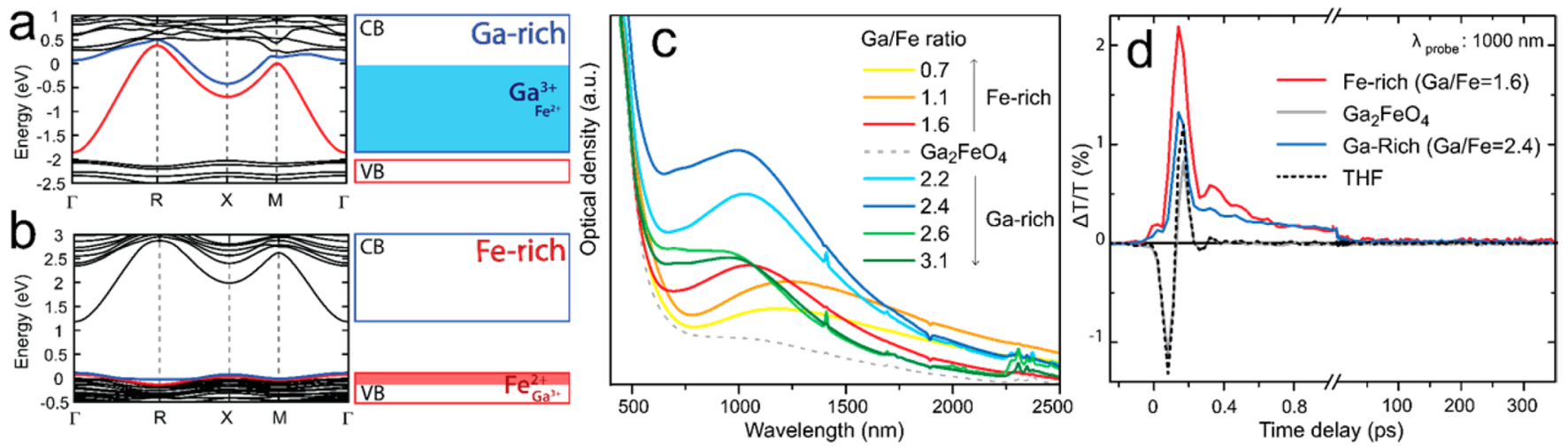

Figure 2. (a,b) DFT band structure calculations along the $\Gamma-\mathrm{R}-\mathrm{X}-\mathrm{M}-\Gamma$ cubic symmetry line of Ga-rich (a) and Fe-rich (b) GFO systems. The highest occupied band (VBM) and lowest unoccupied band (CBM) are colored in red and blue, respectively. (c) Optical properties of Ga-rich and Fe-rich GFO NCs. UV-VIS-NIR absorption curves of solutions of GFO NCs at different Fe and Ga stoichiometries, dispersed in TCE and normalized at $400 \mathrm{~nm}$. (d) Differential transmission dynamics at $1000 \mathrm{~nm}$ for Ga-rich $(\mathrm{Ga} / \mathrm{Fe}=2.4$, blue solid line), Fe-rich $(\mathrm{Ga} / \mathrm{Fe}=1.6$, red solid line), and stoichiometric GFO NCs (gray solid line); the black dotted line shows the dynamic for the solvent, THF.

ICP elemental analysis, confirming the near-stoichiometric nature of our NCs. This crystal structure is characterized by a face-centered cubic lattice of oxide ions that generates both $\mathrm{O}_{h}$ and $\mathrm{T}_{\mathrm{d}}$ sites. $\mathrm{Ga}^{3+}$ ions are equally distributed between $\mathrm{O}_{h}$ and $\mathrm{T}_{\mathrm{d}}$ sites while $\mathrm{Fe}^{2+}$ cations reside on $\mathrm{O}_{\mathrm{h}}$ sites. The overall structure can be written, then, as spinel $(\mathrm{Ga})^{\mathrm{T}_{\mathrm{d}}}(\mathrm{FeGa})^{\mathrm{O}_{\mathrm{h}}} \mathrm{O}_{4}$ (see Figure 1c) ${ }^{61}$ Due to the lack of comprehensive data about the electronic properties of $\mathrm{Ga}_{2} \mathrm{FeO}_{4}$, we performed DFT calculations in order to elucidate the band structure of such material. Within the level of modeling used, stoichiometric GFO displays a direct band gap of $0.3 \mathrm{eV}$ at $\Gamma$, featuring a flat $\mathrm{Fe}_{\mathrm{d}} / \mathrm{O}_{\mathrm{p}}$ derived VBM and a rather dispersive $\mathrm{Ga}_{\mathrm{p}} / \mathrm{Ga}_{\mathrm{s}} / \mathrm{O}_{\mathrm{p}} / \mathrm{O}_{\mathrm{s}}$ derived CBM (see Figure 1d).

The UV-vis-NIR absorption curve of GFO NCs featured an absorption edge with an onset at about $700 \mathrm{~nm}$ and a broad shoulder peaked at $1150 \mathrm{~nm}$ which tails down to $2000 \mathrm{~nm}$ (see Figure 2c, dashed gray line). In order to explain such optical features, we performed DFT calculations and we computed the energy-dependent density of transitions (joint density of states, or jdos), from 950 to $\sim 4000 \mathrm{~nm}$ (see Figure S1 of the SI). A first weak peak at about $0.4 \mathrm{eV}(\sim 3100 \mathrm{~nm})$ followed by a steep rise with a first maximum at $\sim 1.1 \mathrm{eV}$ $(\sim 1130 \mathrm{~nm})$ were clearly distinguished in the jdos plot. Using the $\mathrm{ab}$ initio computed dielectric functions of the stoichiometric and the off-stoichiometric GFO materials, we simulated, using the Mie theory, the absorption profile. The latter, indeed, was characterized by both a weak peak at $1150 \mathrm{~nm}$ and a steep absorption onset at $\sim 700 \mathrm{~nm}$ as the experimental optical density (see Figure S1 of the SI). The peak at $1150 \mathrm{~nm}$ was, thus, assigned to the large number of transitions connecting the center of the $\mathrm{Fe}_{\mathrm{d}} / \mathrm{O}_{\mathrm{p}}$ derived band at $\left(E_{\mathrm{f}}-0.8\right) \mathrm{eV}$ and the onset of the conduction band at $\left(E_{\mathrm{f}}+0.3\right) \mathrm{eV}$, with $E_{\mathrm{f}}$ being the Fermi level, as evinced by the analysis of the jdos plot. On the other hand, the steep rise at $\sim 700 \mathrm{~nm}$ was rationalized by the abrupt increase of the DFT density of states observed at $\left(\sim E_{\mathrm{f}}+\right.$ 2) $\mathrm{eV}(\sim 620 \mathrm{~nm})$ (see Figure 1d).

According to the theoretical predictions by Paudel et al., ${ }^{57}$ intrinsic $\mathrm{n}$ - or $\mathrm{p}$-doping in spinel oxides can be achieved by the generation of antisite defects and/or off-stoichiometry. In the specific case of GFO material, it was calculated that a $\mathrm{Ga}^{3+}$ ion substituting a $\mathrm{Fe}^{2+}$ cation $\left(\mathrm{Ga}_{\mathrm{Fe} 2+}^{3+}\right)$ should create a donor level close to the $\mathrm{CBM}$, while a $\mathrm{Fe}^{2+}$ cation replacing a $\mathrm{Ga}^{3+}$ one $\left(\mathrm{Fe}_{\mathrm{Ga} 3+}^{2+}\right)$ should produce a shallow acceptor level. ${ }^{57}$ The actual modification of the $\mathrm{Ga}_{2} \mathrm{FeO}_{4}$ band structure in off-stoichiometric conditions and the exact energy levels of antisite defects were further investigated by us, using DFT calculations. Given the strong tendency of $\mathrm{Ga}^{3+}$ ions to tetrahedrally coordinate with $\mathrm{O}^{2-}$ anions and of $\mathrm{Fe}^{2+}$ cations to prefer $\mathrm{O}_{h}$ sites, the cross substitution of $\mathrm{Ga}^{3+}$ and $\mathrm{Fe}^{2+}$ ions was assumed to take place in $\mathrm{O}_{\mathrm{h}}$ sites. ${ }^{68-70}$ For the present study, as our off-stoichiometric GFO NCs can be considered as heavily doped semiconductors, the hybridization of the aforementioned acceptor and donor levels form dispersive bands, as displayed in Figure 2a,b. More precisely, in Ga-rich conditions a n-type doping emerged as an effect of the partial population of the dispersive CBM (light blue area in Figure 2a, right panel) which gives the material a metallic character. A similar behavior was also observed in Ferich conditions (red area the right panel of Figure 2b). However, in the latter case, the lowest unoccupied band is flat (see Figure S2c of the SI), while the highest occupied band has a larger curvature, leading to effective electron and hole masses of $14.3 m_{\mathrm{e}}$ and $3.9 m_{\mathrm{e}}\left(m_{\mathrm{e}}\right.$ : electron mass $)$, respectively. The different mobility of electrons and holes suggests a mechanism of p-type doping in Fe-rich samples.

In order to induce off-stoichiometry in our system, and thus to generate doping, we performed the synthesis of GFO NCs using either Ga-rich conditions $(\mathrm{Ga} / \mathrm{Fe}$ precursors ratio above 2) or Fe-rich conditions ( $\mathrm{Ga} / \mathrm{Fe}$ precursors ratio below 1.5). The $\mathrm{Ga} / \mathrm{Fe}$ ratio of each GFO NC sample was closely related to the $\mathrm{Ga} / \mathrm{Fe}$ precursors ratio used in its synthesis, as measured by ICP elemental analysis (see Table 1 in the Experimental Section). The XRD analysis evidenced that all the off-

Table 1. Experimental Ga/Fe Precursors Ratios and the Composition of the Corresponding GFO NCs

$\begin{array}{lcc} & \mathrm{Ga} / \mathrm{Fe} \text { feed ratio } & \mathrm{Ga} / \mathrm{Fe} \text { ratio in } \mathrm{NCs}^{a} \\ \text { Fe-rich } & 0.7 & 0.5 \\ & 1 & 1.1 \\ & 1.4 & 1.6 \\ \text { stoichiometric } & 2 & 1.9 \\ \text { Ga-rich } & 2.2 & 2.2 \\ & 2.4 & 2.4 \\ & 2.8 & 2.6 \\ & 3 & 3.1\end{array}$

${ }^{a_{T}} \mathrm{The} \mathrm{Ga} / \mathrm{Fe}$ ratios were measured via ICP elemental analysis. 
stoichiometric samples had an inverse-spinel $\mathrm{Ga}_{2} \mathrm{FeO}_{4}$-like structure with the same lattice parameters of the stoichiometric GFO NCs (see Figure S3c of the SI). Also, the size, size distribution and shape of Ga-rich and Fe-rich GFO NCs were similar to those of the corresponding stoichiometric NCs sample (see Figure S3a,b of the SI).

The UV-vis-NIR absorption curves of off-stoichiometric samples, reported in Figure 2c, were all characterized by an intense band in the NIR region. In the case of Ga-rich series (blue-green curves), such band shifted from $1030 \mathrm{~nm}(\mathrm{Ga} / \mathrm{Fe}=$ 2.2) to $995 \mathrm{~nm}(\mathrm{Ga} / \mathrm{Fe}=2.4)$ increasing in intensity (see Figure $2 c$, green curves). A further incorporation of gallium in the NCs $(\mathrm{Ga} / \mathrm{Fe} \geq 2.6)$ led to a drop of the NIR absorption band. Similarly, in the Fe-rich series, we observed that the band peaking at $1050 \mathrm{~nm}$ (for $\mathrm{Ga} / \mathrm{Fe}=1.6)$ systematically dropped in intensity and red-shifted at increasing $\mathrm{Fe} / \mathrm{Ga}$ molar ratios $(\mathrm{Ga} / \mathrm{Fe} \leq 1.1)$ (see Figure $2 \mathrm{c}$, yellow, orange, and red curves).

The nature of such pronounced NIR absorption bands, that characterize the off-stoichiometric GFO NCs, was investigated through pump-probe experiments on three representative samples: the stoichiometric $\mathrm{Ga}_{2} \mathrm{FeO}_{4} \mathrm{NCs}$ sample, a Ga-rich $(\mathrm{Ga} / \mathrm{Fe}=2.4)$ sample, and a Fe-rich $(\mathrm{Ga} / \mathrm{Fe}=1.6)$ sample. Pump-probe spectroscopy is a powerful tool to study the characteristic carrier dynamics and to give insight into the physical nature of the optical resonance. The samples, dispersed in THF, were analyzed using a pump pulse at $800 \mathrm{~nm}$ to excite the NIR resonance with moderate pump powers $(1.6 \mu \mathrm{J}$ as energy per pulse) and a pulse in the NIR region to probe the behavior of the NIR resonance. In Figure $2 \mathrm{~d}$ are given the temporal dynamics for both samples at $1000 \mathrm{~nm}$ (Ga-rich, blue curve and Fe-rich, red curve), together with the stoichiometric $\mathrm{Ga}_{2} \mathrm{FeO}_{4}$ sample. We provide in Figure $2 \mathrm{~d}$ also the measurement on THF alone as a reference, which displays the temporal resolution of our measurements. A nonlinearity was observed in the transient spectra of both Ga- and Fe-rich samples, with a bleach signal in the region of the NIR resonances. An initial very fast decay, recovering in less than $1 \mathrm{ps}$, was observed in both off-stoichiometric GFO samples, followed by a second much slower decay of a couple of hundred picoseconds (please note the break in the time axis). The two-step temporal evolution is typical for plasmonic resonances, where the initial fast decay is ascribed to the cooling of the excited carrier gas via the interaction with the lattice, while the second slower decay is assigned to the cooling of the lattice through the emission of phonons to the surrounding medium. ${ }^{71}$ The response of Ferich GFO NCs was fitted by a biexponential decay that was associated with electron-phonon relaxation $(\sim 1.1 \mathrm{ps})$ and phonon-phonon coupling ( $\sim 223$ ps) processes, as shown in Figures 2d, S4, and S5. Similarly, Ga-rich samples exhibited an electron-phonon relaxation (with a decay time of $840 \mathrm{fs}$ ) and a phonon-phonon coupling (with a decay of 213 ps) (see Figures $2 \mathrm{~d}$ and S4). Similarly to what observed in other doped semiconductors, the electron-phonon relaxation time in our GFO NC is faster than that observed in noble metals as a result of the much lower carrier density and the lower heat capacity of the carriers. ${ }^{72,73}$ These results are consistent with the presence of a LSPR in the NIR for both Fe-rich and Ga-rich GFO NCs. On the other hand, stoichiometric GFO NCs did not show any kind of decay dynamics (see Figures $2 \mathrm{~d}$ and S4). This supports that the broad absorption feature peaked at $1150 \mathrm{~nm}$, that characterizes the steady state absorption of stoichiometric GFO NCs, can be ascribed to interband transitions, as inferred by DFT calculations.
In order to quantitatively describe the NIR response of the free carriers in our NCs and to extract the carrier density $n$, we fitted the LSPR of off-stoichiometric GFO samples. We employed the quasi-static approximation of the Mie theory, according to which the absorption cross section $\sigma_{\mathrm{A}}(\omega)$ can be expressed as

$$
\sigma_{\mathrm{A}}(\omega)=\frac{4 \pi \omega}{c} \sqrt{\varepsilon_{\mathrm{H}}} R^{3} \operatorname{imag}\left(\frac{\varepsilon_{\mathrm{p}(\omega)}-\varepsilon_{\mathrm{H}}}{\varepsilon_{\mathrm{p}(\omega)}+2 \varepsilon_{\mathrm{H}}}\right)
$$

where $\varepsilon_{\mathrm{H}}$ depicts the dielectric constant of the solvent, surrounding the NCs, $\varepsilon_{\mathrm{p}}(\omega)$ is the frequency dependent dielectric function of the material, $R$ is the NC radius, $c$ is the speed of light, and $\omega$ is the optical frequency. The absorption of the NC solution could be calculated with the Lambert-Beer law according to

$$
A=\frac{N \sigma_{\mathrm{A}} L}{\log (10)}
$$

where $N$ is the number density of NCs in solution (a parameter that was fitted in our case) and $L$ is the path length of the cuvette. To describe the NIR response of the free carriers in our NCs, we employed the Drude dielectric function $\varepsilon_{\mathrm{p}}(\omega)$ :

$$
\varepsilon_{\mathrm{p}}(\omega)=\varepsilon_{\infty}-\frac{\omega_{\mathrm{p}}^{2}}{\left(\omega^{2}+i \omega \gamma\right)}
$$

where $\varepsilon_{\infty}$ depicts the high frequency dielectric constant, $\gamma$ is the free carrier damping constant, and $\omega_{\mathrm{p}}$ is the plasma frequency given as

$$
\omega_{\mathrm{p}}=\sqrt{\frac{n e^{2}}{\varepsilon_{0} m^{*}}}
$$

Here, $e$ is the electron charge, $m^{*}$ is the effective mass, $\varepsilon_{0}$ is the vacuum dielectric permittivity, and $n$ is the carrier density. As no $\varepsilon_{\infty}$ values for spinel-type oxide $\mathrm{Ga}_{2} \mathrm{FeO}_{4}$ are reported in the literature, they were extracted from the dielectric functions computed with DFT, obtaining 3.74 and 4.56 in the case of Garich and Fe-rich samples, respectively. The resulting fits results are shown in Figure 3.

From these fits, assuming that free carriers are electrons in Ga-rich samples, with $m^{*}=m_{\mathrm{e}}$, and holes in Fe-rich NCs, with
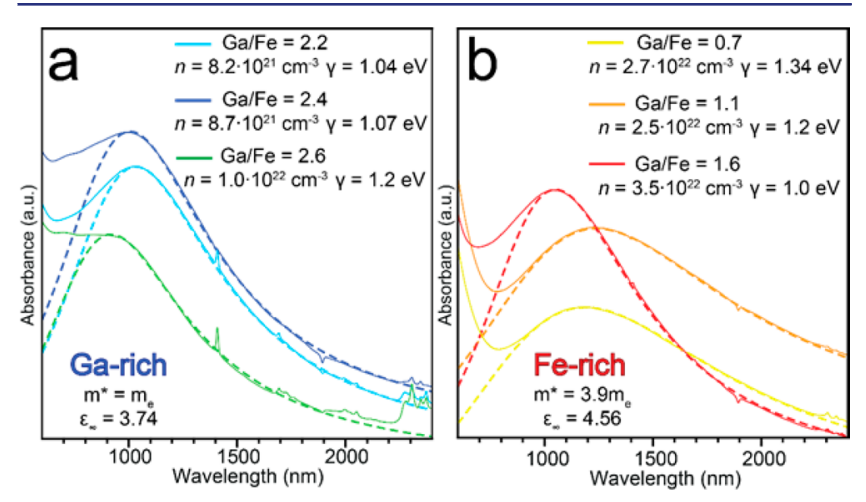

Figure 3. Experimental absorption spectra of solutions of Ga-rich (a) and Fe-rich (b) GFO NCs together with their fit that was obtained by employing the Mie theory and the Drude model. The values of the electron and hole effective mass $m^{*}$ as well as the high frequency dielectric constant $\varepsilon_{\infty}$ have been extracted from the dielectric function computed with DFT. 
$m^{*}=3.9 m_{\mathrm{e}}$, as previously calculated by DFT, we extracted the carrier density (see Figure 3 ). In all the off-stoichiometric samples the concentration of free carriers was found to be in the order of $10^{22} \mathrm{~cm}^{-3}$, which is in the range of typical other degenerately doped semiconductors. ${ }^{8}$ Notably, our fits with the Drude model showed that the damping constant, $\gamma$, increased dramatically the more diverging from the $\mathrm{Ga}_{2} \mathrm{FeO}_{4}$ stoichiometry (see Figures 3 and S6). The intrinsic doping of GFO NCs, thus, seems to be efficient only in a specific stoichiometry range, i.e., $1.6 \leq \mathrm{Ga} / \mathrm{Fe} \leq 2.4$, outside which, charge compensation phenomena appear to have a detrimental effect on the LSPR. This has been rationalized by the analysis of the DFT results. In the case of Ga-rich samples, for example, by replacing one $\mathrm{Fe}^{2+}$ ion with one $\mathrm{Ga}^{3+}$ ion (with a resulting $\mathrm{Ga}$ / Fe ratio of 2.4), the dispersive conduction band becomes halffilled giving rise to a strongly metallic system (see Figure S2a of the SI). If two $\mathrm{Ga}^{3+}$ cations replace two $\mathrm{Fe}^{2+}$ ions (i.e., $\mathrm{Ga} / \mathrm{Fe}=$ 3 ), the dispersive conduction band becomes filled, giving rise to a semimetallic system (see Figure S2b of the SI), damping the optical response.

It is important to underline that the extracted values of free carrier densities $\left(10^{22} \mathrm{~cm}^{-3}\right)$ appear to be high, given that a theoretical limit of carriers added to the system leads to $10^{21}$ (1 or 2 dopants per unit cell). Also, the LSPR bands seem to be quite broad, with consequent high damping factors. These features can be both rationalized considering that the LSPR is largely overlapping with interband transitions of the GFO material in the NIR. This leads not only to an additional loss mechanism of the LSPR as it is well-known also in gold, but it additionally limits the applicability of the Drude model in this range. ${ }^{5}$ Nevertheless, our results can serve as a first estimation, while a more precise evaluation of the exact carrier density remains for future works.

To qualitatively elucidate the actual sign of the majority carriers responsible for the above presented LSPR features, we carried out a series of transient photovoltage (PV) measurements in a three-electrode electrochemical cell. To this purpose, four mesoporous films were prepared using different GFO samples: stoichiometric $\mathrm{Ga}_{2} \mathrm{FeO}_{4}$, one batch of Ga-rich NCs $(\mathrm{Ga} / \mathrm{Fe}=2.4)$ and two batches of Fe-rich NCs $(\mathrm{Ga} / \mathrm{Fe}=$ 1.6 and 1.1). Each sample was turned into a viscous screenprintable paste, deposited on a glass substrate and then subjected to a thermal annealing in air at $390{ }^{\circ} \mathrm{C}$ (see the Experimental Section for details). The resulting electrodes were immersed in a suitable electrolytic solution and exposed to white light irradiation: the sign of the photogenerated potential revealed the sign of the majority charge carriers.

When a semiconductor is immersed in an electrolyte, an electric current flows across the junction until electronic equilibrium is reached, a situation in which the Fermi level is pinned at the semiconductor/electrolyte interface. If the redox potential of the electrolyte $\left(\mathrm{E}_{\text {redox }}\right)$ is located inside the bandgap of the semiconductor, in case of n-type doping the bands of the semiconductor are shifted to more positive potentials with a consequent upward bending, as schematically depicted in Figure $4 \mathrm{a}$, left and central panels (where the energy scale is referred to the Normal Hydrogen Electrode, NHE). ${ }^{62}$ After exposure to white light, the photogenerated minority carriers (holes) move to the surface of the semiconductor where they are compensated by negatively charged species from the electrolyte, thus slightly diminishing the band bending (Figure $4 \mathrm{a}$, right panel) and, consequently, causing the measured potential to move to more negative values (with respect to the
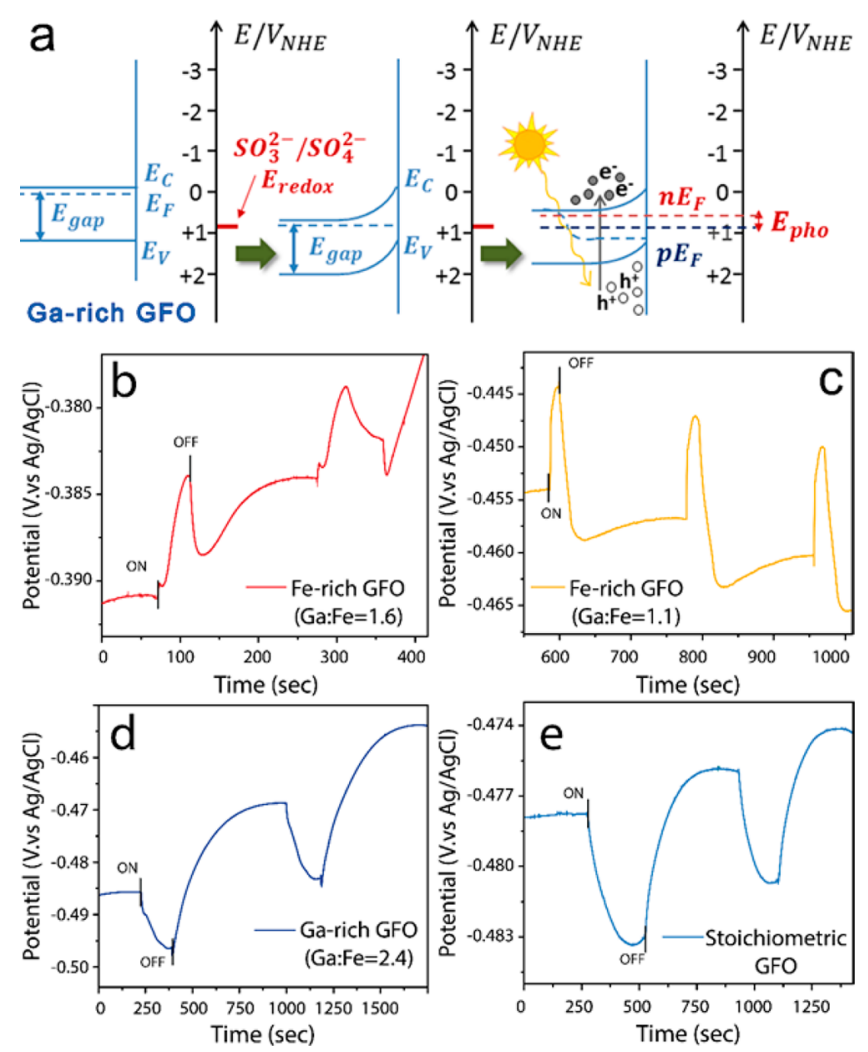

Figure 4. (a) Energy level diagram of a n-type doped semiconductor in a typical cell for PV measurements: before (left) and after (middle) reaching the equilibrium with the electrolyte in the dark, and under illumination (right). The redox potential of the electrolyte $\left(\mathrm{Na}_{2} \mathrm{SO}_{3}\right)$ is indicated with $E_{\text {redox }}$, the quasi-Fermi level for electrons with $n E_{\mathrm{F}}$, for holes with $p E_{\mathrm{F}}$, and the photopotential with $E_{\mathrm{pho}}$. $(\mathrm{b}-\mathrm{e}) \mathrm{PV}$ transients of Fe-rich (b,c), Ga-rich (d), and stoichiometric (e) GFO NCs measured by illuminating the samples with a white light lamp.

$\mathrm{Ag} / \mathrm{AgCl}$ reference electrode). As a reminder for the reader, when a doped semiconductor is illuminated with a radiation above its optical band gap, the concentration of minority carriers increases considerably, while the density of majority carriers can be considered invariant. ${ }^{62}$ In case of a p-type doped semiconductor, the starting band bending occurs in the opposite direction as a consequence of the Fermi energy pinning (the bands are bended downward). After shining light, thus, the measured potential changes to more positive potential (with respect to the $\mathrm{Ag} / \mathrm{AgCl}$ reference electrode).

In our experiments the PV transients of both Fe-rich samples exhibited a positive potential drop upon illumination, pointing to a p-type character (see Figure $4 b, c$ ). On the other hand, negative phototransients were observed for Ga-rich samples, as shown in Figure 4d. The stoichiometric GFO NCs exhibited a n-type character (as the Ga-rich ones), suggesting that a thermally annealed film made of $\mathrm{Ga}_{2} \mathrm{FeO}_{4} \mathrm{NCs}$ behaves as an intrinsic n-type semiconductor (see Figure 4e).

To further support these findings, we also carried out a set of electrochemical impedance spectroscopy (EIS) measurements and, upon extrapolating the values of the inverse of the spacecharge capacitance squared $\left(1 / C_{\mathrm{SC}}{ }^{2}\right)$ at the interface with a 0.5 $\mathrm{M} \mathrm{Na} \mathrm{SO}_{3}$ aqueous solution, we traced the Mott-Schottky plots of the four electrodes (see Figure 5). ${ }^{63,64}$ The intercept of the slope of $C_{\mathrm{SC}}{ }^{-2}$ with the $V$-axis is generally referred as flatband potential $\left(V_{\mathrm{FB}}\right)$ : it provides an approximate estimation of band-bending caused by the Fermi level pinning at the 

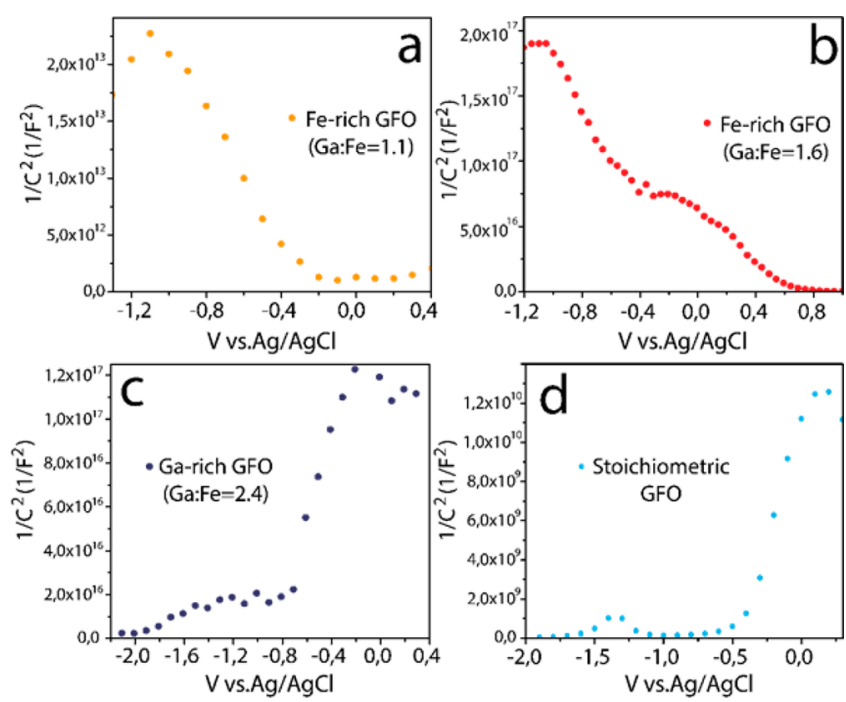

Figure 5. Mott-Schottky plots of four different mesoporous films made with $\mathrm{NCs}$ of GFO measured in an $\mathrm{Na}_{2} \mathrm{SO}_{3}$ electrolyte (at $\mathrm{pH}=$ 9) in dark. The plots were obtained using the values of the space charge region capacity of the semiconductor-electrolyte junction calculated by EIS.

semiconductor/electrolyte interface at equilibrium (see Figure $4 a$, middle panel). ${ }^{62}$ Upon applying an external potential, it is possible to increase or reduce the band bending. In particular, the sign and the intensity of the external potential required to flatten the band are intrinsically correlated with the sign and the density of majority carriers in the semiconductor. Thus, in ndoped semiconductors the flat band condition is achieved at negative potentials. In p-doped semiconductors, on the other hand, this happens at positives potentials. A comparison of the Mott-Schottky plots of these four families of mesoporous films corroborates what found by PV measurements, as, again, an intrinsic p-type conductivity was found for the Fe-rich samples (see Figure 5a,b) and a n-type conductivity for both stoichiometric and Ga-rich GFO NCs (see Figure 5c,d). In particular, the measured value of the intercept of the linear part of $C_{\mathrm{SC}}{ }^{-2}$ with the $x$-axis displayed a univocal trend as it moved from about $0.4-0.6 \mathrm{~V}$ (vs $\mathrm{Ag} / \mathrm{AgCl}$ ) for the $\mathrm{Ga} / \mathrm{Fe}=1.6$ sample to about $-0.7 \mathrm{~V}$ (vs $\mathrm{Ag} / \mathrm{AgCl}$ ) for the $\mathrm{Ga} / \mathrm{Fe}=2.4$ sample. This trend supported, once again, the different semiconductor characters of Fe-rich samples in respect to Garich and stoichiometric GFO ones.

In the light of these results, with the aim to further corroborate the band structure calculated by DFT, we performed XPS analyses on the various GFO NCs samples, by which it is possible, in principle, to elucidate the oxidation state of gallium and iron ions. More specifically, the generation of free holes in Fe-rich samples can only take place if $\mathrm{Fe}^{2+}$ and not $\mathrm{Fe}^{3+}$ ions do substitute gallium cations, while the generation of free electrons in Ga-rich samples occurs if extra gallium ions have a +3 oxidation state. The results of high resolution XPS analyses for selected off-stoichiometric GFO NC samples are presented in Figure 6.

As it is possible to appreciate in the left panels of Figure 6, in all the off-stoichiometric GFO samples, the Ga $2 \mathrm{p}_{3 / 2}$ peak was at a binding energy of $1117.2 \pm 0.3 \mathrm{eV}$, consistent with a +3 oxidation state. ${ }^{74}$ Conversely, the shape of Fe $2 p$ peaks changed when going from Ga-rich to Fe-rich conditions (see right panels of Figure 6). In Ga-rich conditions, the position and shape of

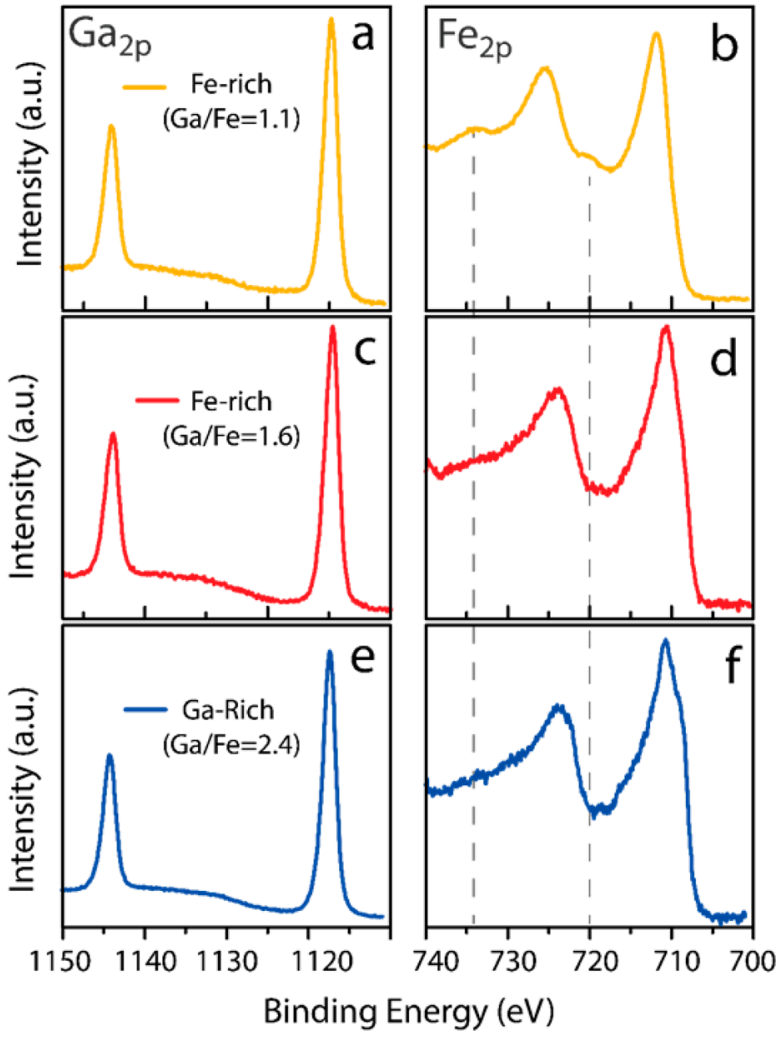

Figure 6. High resolution XPS spectra of two Fe-rich $(\mathrm{Ga} / \mathrm{Fe}=1.1$ and 1.6, orange and red curves, respectively) and a Ga-rich $(\mathrm{Ga} / \mathrm{Fe}=$ 2.4, blue curves) GFO NC samples in the region of the Ga $2 p$ level $(\mathrm{a}, \mathrm{c}, \mathrm{e})$ and $\mathrm{Fe} 2 \mathrm{p}$ level $(\mathrm{b}, \mathrm{d}, \mathrm{f})$. The two vertical dashed lines crossing (b), (d), and (f) panels evidence the position of the shakeup satellite peaks associated with $\mathrm{Fe}^{3+}$ species.

Fe $2 p_{3 / 2}$ and $2 p_{1 / 2}$ peaks were in agreement with those reported in the literature for $\mathrm{Fe}^{2+}$ compounds. ${ }^{75,76} \mathrm{~A}$ similar Fe $2 \mathrm{p}$ signal profile was seen in the $\mathrm{Ga} / \mathrm{Fe}=1.6$ sample, suggesting that also in these GFO NCs $\mathrm{Fe}$ is present mainly as $\mathrm{Fe}^{2+}$. Conversely, in the $\mathrm{Ga} / \mathrm{Fe}=1.1$ sample shakeup satellite peaks appeared at 720 and $734 \mathrm{eV}$ (located approximately at binding energies $8 \mathrm{eV}$ higher than the main XPS peaks), which can be ascribed to the $\mathrm{Fe}^{3+}$ species (see Figure $6 \mathrm{~b}$ ). ${ }^{77}$

These findings suggest that in Ga-rich conditions $\mathrm{Ga}^{3+}$ ions do replace $\mathrm{Fe}^{2+}$ ones in GFO NCs, eventually leading to n-type doping. On the other hand, in Fe-rich conditions $\mathrm{Fe}^{2+}$ substitute $\mathrm{Ga}^{3+}$ cations up to a stoichiometry of $\mathrm{Ga} / \mathrm{Fe}=1.6$ with the consequent effective injection of free holes (i.e., p-type doping). The charge imbalance generated by a further increase in iron content in GFO NCs is compensated by the oxidation of part of $\mathrm{Fe}$ ions from +2 to +3 . This scenario would also explain the less efficient injection of free carriers (i.e., lower LSPR response) observed when increasing the concentration of iron in GFO NCs above $\mathrm{Ga} / \mathrm{Fe}=1.6$.

\section{CONCLUSIONS}

In summary, we have developed a colloidal synthesis of GFO NCs having a diameter of $\sim 5 \mathrm{~nm}$ and an inverse spinel-type crystal structure. By tuning the relative amount of gallium and iron precursors we could prepare both stoichiometric and offstoichiometric (Fe-rich and Ga-rich) GFO samples. Offstoichiometric GFO NCs exhibited a LSPR peaked around $1000 \mathrm{~nm}$ in the NIR, as confirmed by pump-probe 
spectroscopy analysis. Photoelectrochemical analyses indicated that the free carriers responsible for such LSPR are holes in case of Fe-rich samples and electrons in Ga-rich NCs. DFT calculations elucidated our findings, more specifically: (i) the replacement of a fraction of the $\mathrm{Fe}^{2+}$ ions with $\mathrm{Ga}^{3+}$ cations (that takes place in Ga-rich conditions) is compensated by the generation of free electrons, which populate the dispersive CBM of GFO; (ii) in Fe-rich conditions, a fraction of $\mathrm{Ga}^{3+}$ ions is substituted by $\mathrm{Fe}^{2+}$ cations with the consequent formation of free "heavy" holes in the VBM. The off-stoichiometric range in which GFO NCs were found to be efficiently doped was found to be $1.6 \leq \mathrm{Ga} / \mathrm{Fe} \leq 2.4$. We believe that our results, which underline the bipolar nature of gallium iron oxide, will strengthen the interest in spinel-type oxide materials for applications as p-type transparent conducive materials and in plasmonics.

\section{ASSOCIATED CONTENT}

\section{S Supporting Information}

The Supporting Information is available free of charge on the ACS Publications website at DOI: 10.1021/jacs.6b11063.

Computed joint density of states, DFT band structure calculations, TEM and XRD characterizations of offstoichiometric GFO NCs, differential transmission dynamics of a Fe-rich ( $\mathrm{Ga} / \mathrm{Fe}$ ratio of 1.1) GFO sample, differential transmission spectra of different GFO samples, fitting of the experimental absorption spectra, XRD and SEM characterizations of mesoporous films used for photovoltage measurements (PDF)

\section{AUTHOR INFORMATION}

\section{Corresponding Authors}

*luca.detrizio@iit.it

*liberato.manna@iit.it

ORCID ${ }^{\circledR}$

Margherita Zavelani-Rossi: 0000-0001-9910-0391

Luca De Trizio: 0000-0002-1514-6358

Notes

The authors declare no competing financial interest.

\section{ACKNOWLEDGMENTS}

We acknowledge funding from the European Union under Grant Agreement No. 614897 (ERC Grant TRANS-NANO). Antonio Qualtieri is acknowledged for help with the acquisition of SEM images. The IIT platform CompuNet and the Italian Supercomputer Center CINECA (Bologna, Italy) are acknowledged for the computational resources.

\section{REFERENCES}

(1) Schimpf, A. M.; Thakkar, N.; Gunthardt, C. E.; Masiello, D. J.; Gamelin, D. R. ACS Nano 2014, 8, 1065-1072.

(2) Lounis, S. D.; Runnerstrom, E. L.; Llordés, A.; Milliron, D. J. J. Phys. Chem. Lett. 2014, 5, 1564-1574.

(3) Agrawal, A.; Kriegel, I.; Milliron, D. J. J. Phys. Chem. C 2015, 119, 6227-6238.

(4) Comin, A.; Manna, L. Chem. Soc. Rev. 2014, 43, 3957-3975.

(5) Naik, G. V.; Shalaev, V. M.; Boltasseva, A. Adv. Mater. 2013, 25, 3264-3294.

(6) Noginov, M. A.; Gu, L.; Livenere, J.; Zhu, G.; Pradhan, A. K.; Mundle, R.; Bahoura, M.; Barnakov, Y. A.; Podolskiy, V. A. Appl. Phys. Lett. 2011, 99, 021101.

(7) Routzahn, A. L.; White, S. L.; Fong, L.-K.; Jain, P. K. Isr. J. Chem. 2012, 52, 983-991.
(8) Liu, X.; Swihart, M. T. Chem. Soc. Rev. 2014, 43, 3908-3920.

(9) Tandon, B.; Ashok, A.; Nag, A. Pramana 2015, 84, 1087-1098.

(10) Faucheaux, J. A.; Stanton, A. L. D.; Jain, P. K. J. Phys. Chem. Lett. 2014, 5, 976-985.

(11) Schimpf, A. M.; Lounis, S. D.; Runnerstrom, E. L.; Milliron, D. J.; Gamelin, D. R. J. Am. Chem. Soc. 2015, 137, 518-524.

(12) Kriegel, I.; Rodríguez-Fernández, J.; Wisnet, A.; Zhang, H.; Waurisch, C.; Eychmüller, A.; Dubavik, A.; Govorov, A. O.; Feldmann, J. ACS Nano 2013, 7, 4367-4377.

(13) Zhao, Y.; Pan, H.; Lou, Y.; Qiu, X.; Zhu, J.; Burda, C. J. Am. Chem. Soc. 2009, 131, 4253-4261.

(14) Kriegel, I.; Jiang, C.; Rodríguez-Fernández, J.; Schaller, R. D.; Talapin, D. V.; da Como, E.; Feldmann, J. J. Am. Chem. Soc. 2012, 134, $1583-1590$

(15) De Trizio, L.; Gaspari, R.; Bertoni, G.; Kriegel, I.; Moretti, L.; Scotognella, F.; Maserati, L.; Zhang, Y.; Messina, G. C.; Prato, M.; Marras, S.; Cavalli, A.; Manna, L. Chem. Mater. 2015, 27, 1120-1128.

(16) Zhou, S.; Ni, Z.; Ding, Y.; Sugaya, M.; Pi, X.; Nozaki, T. ACS Photonics 2016, 3, 415-422.

(17) Garcia, G.; Buonsanti, R.; Runnerstrom, E. L.; Mendelsberg, R. J.; Llordes, A.; Anders, A.; Richardson, T. J.; Milliron, D. J. Nano Lett. 2011, 11, 4415-4420.

(18) Stadler, A. Materials 2012, 5, 661-683.

(19) Li, H.; Winget, P.; Brédas, J.-L. Chem. Mater. 2014, 26, 631646.

(20) Mendelsberg, R. J.; McBride, P. M.; Duong, J. T.; Bailey, M. J.; Llordes, A.; Milliron, D. J.; Helms, B. A. Adv. Opt. Mater. 2015, 3, 1293-1300.

(21) Abb, M.; Wang, Y.; Papasimakis, N.; de Groot, C. H.; Muskens, O. L. Nano Lett. 2014, 14, 346-352.

(22) Llordes, A.; Garcia, G.; Gazquez, J.; Milliron, D. J. Nature 2013, 500, 323-326.

(23) Naik, G. V.; Liu, J.; Kildishev, A. V.; Shalaev, V. M.; Boltasseva, A. Proc. Natl. Acad. Sci. U. S. A. 2012, 109, 8834-8838.

(24) Ohodnicki, P. R., Jr; Wang, C.; Andio, M. Thin Solid Films 2013, $539,327-336$.

(25) Fortunato, E.; Barquinha, P.; Martins, R. Adv. Mater. 2012, 24, 2945-2986.

(26) Ohta, H.; Hosono, H. Mater. Today 2004, 7, 42-51.

(27) Robertson, J.; Clark, S. J. Phys. Rev. B: Condens. Matter Mater. Phys. 2011, 83, 075205.

(28) Schimpf, A. M.; Knowles, K. E.; Carroll, G. M.; Gamelin, D. R. Acc. Chem. Res. 2015, 48, 1929-1937.

(29) Manthiram, K.; Alivisatos, A. P. J. Am. Chem. Soc. 2012, 134, 3995-3998.

(30) Huang, Q.; Hu, S.; Zhuang, J.; Wang, X. Chem. - Eur. J. 2012, 18, $15283-15287$.

(31) Choi, S.-I.; Nam, K. M.; Park, B. K.; Seo, W. S.; Park, J. T. Chem. Mater. 2008, 20, 2609-2611.

(32) Luo, S.; Yang, D.; Feng, J.; Ng, K. M. J. Nanopart. Res. 2014, 16, $1-12$.

(33) Kanehara, M.; Koike, H.; Yoshinaga, T.; Teranishi, T. J. Am. Chem. Soc. 2009, 131, 17736-17737.

(34) Della Gaspera, E.; Chesman, A. S. R.; van Embden, J.; Jasieniak, J. J. ACS Nano 2014, 8, 9154-9163.

(35) Buonsanti, R.; Llordes, A.; Aloni, S.; Helms, B. A.; Milliron, D. J. Nano Lett. 2011, 11, 4706-4710.

(36) zum Felde, U.; Haase, M.; Weller, H. J. Phys. Chem. B 2000, 104, 9388-9395.

(37) Gordon, T. R.; Paik, T.; Klein, D. R.; Naik, G. V.; Caglayan, H.; Boltasseva, A.; Murray, C. B. Nano Lett. 2013, 13, 2857-2863.

(38) Hamza, M. K.; Bluet, J. M.; Masenelli-Varlot, K.; Canut, B.; Boisron, O.; Melinon, P.; Masenelli, B. Nanoscale 2015, 7, 1203012037.

(39) Runnerstrom, E. L.; Bergerud, A.; Agrawal, A.; Johns, R. W.; Dahlman, C. J.; Singh, A.; Selbach, S. M.; Milliron, D. J. Nano Lett. 2016, 16, 3390-3398. 
(40) De Trizio, L.; Buonsanti, R.; Schimpf, A. M.; Llordes, A.; Gamelin, D. R.; Simonutti, R.; Milliron, D. J. Chem. Mater. 2013, 25, 3383-3390.

(41) Mattox, T. M.; Bergerud, A.; Agrawal, A.; Milliron, D. J. Chem. Mater. 2014, 26, 1779-1784.

(42) Zhao, Z.; Yin, S.; Guo, C.; Sato, T. J. Nanosci. Nanotechnol. 2015, 15, 7173-7176.

(43) Ye, X.; Fei, J.; Diroll, B. T.; Paik, T.; Murray, C. B. J. Am. Chem. Soc. 2014, 136, 11680-11686.

(44) Quackenbush, N. F.; Allen, J. P.; Scanlon, D. O.; Sallis, S.; Hewlett, J. A.; Nandur, A. S.; Chen, B.; Smith, K. E.; Weiland, C.; Fischer, D. A.; Woicik, J. C.; White, B. E.; Watson, G. W.; Piper, L. F. J. Chem. Mater. 2013, 25, 3114-3123.

(45) Kawazoe, H.; Yanagi, H.; Ueda, K.; Hosono, H. MRS Bull. 2000, $25,28-36$.

(46) Nomura, K.; Ohta, H.; Ueda, K.; Kamiya, T.; Hirano, M.; Hosono, H. Science 2003, 300, 1269-1272.

(47) Bhatia, A.; Hautier, G.; Nilgianskul, T.; Miglio, A.; Sun, J.; Kim, H. J.; Kim, K. H.; Chen, S.; Rignanese, G.-M.; Gonze, X.; Suntivich, J. Chem. Mater. 2016, 28, 30-34.

(48) Wang, Z.; Nayak, P. K.; Caraveo-Frescas, J. A.; Alshareef, H. N. Adv. Mater. 2016, 28, 3831-3892.

(49) Sheng, S.; Fang, G.; Li, C.; Xu, S.; Zhao, X. Phys. Status Solidi A 2006, 203, 1891-1900.

(50) Zunger, A. Appl. Phys. Lett. 2003, 83, 57-59.

(51) Kawazoe, H.; Yasukawa, M.; Hyodo, H.; Kurita, M.; Yanagi, H.; Hosono, H. Nature 1997, 389, 939-942.

(52) Scanlon, D. O.; Watson, G. W. J. Mater. Chem. 2011, 21, 36553663.

(53) Snure, M.; Tiwari, A. Appl. Phys. Lett. 2007, 91, 092123.

(54) Ogo, Y.; Hiramatsu, H.; Nomura, K.; Yanagi, H.; Kamiya, T.; Kimura, M.; Hirano, M.; Hosono, H. Phys. Status Solidi A 2009, 206, 2187-2191.

(55) Caraveo-Frescas, J. A.; Nayak, P. K.; Al-Jawhari, H. A.; Granato, D. B.; Schwingenschlögl, U.; Alshareef, H. N. ACS Nano 2013, 7, 5160-5167.

(56) Shi, Y.; Ndione, P. F.; Lim, L. Y.; Sokaras, D.; Weng, T.-C.; Nagaraja, A. R.; Karydas, A. G.; Perkins, J. D.; Mason, T. O.; Ginley, D. S.; Zunger, A.; Toney, M. F. Chem. Mater. 2014, 26, 1867-1873.

(57) Paudel, T. R.; Zakutayev, A.; Lany, S.; d'Avezac, M.; Zunger, A. Adv. Funct. Mater. 2011, 21, 4493-4501.

(58) Amini, M. N.; Dixit, H.; Saniz, R.; Lamoen, D.; Partoens, B. Phys. Chem. Chem. Phys. 2014, 16, 2588-2596.

(59) Zakutayev, A.; Paudel, T. R.; Ndione, P. F.; Perkins, J. D.; Lany, S.; Zunger, A.; Ginley, D. S. Phys. Rev. B: Condens. Matter Mater. Phys. 2012, 85, 085204.

(60) Perkins, J. D.; Paudel, T. R.; Zakutayev, A.; Ndione, P. F.; Parilla, P. A.; Young, D. L.; Lany, S.; Ginley, D. S.; Zunger, A.; Perry, N. H.; Tang, Y.; Grayson, M.; Mason, T. O.; Bettinger, J. S.; Shi, Y.; Toney, M. F. Phys. Rev. B: Condens. Matter Mater. Phys. 2011, 84, 205207.

(61) Burnett, D. L.; Harunsani, M. H.; Kashtiban, R. J.; Playford, H. Y.; Sloan, J.; Hannon, A. C.; Walton, R. I. J. Solid State Chem. 2014, 214, 30-37.

(62) Bard, A. J.; Faulkner, L. R. Electrochemical Methods: Fundamentals and Applications; John Wiley \& Sons: Somerset, NJ, 2000.

(63) Orazem, M. E.; Tribollet, B. Electrochemical Impedance Spectroscopy; John Wiley \& Sons: Hoboken, NJ, 2011.

(64) Sellers, M. C. K.; Seebauer, E. G. Thin Solid Films 2011, 519, 2103-2110.

(65) Perdew, J. P.; Burke, K.; Ernzerhof, M. Phys. Rev. Lett. 1996, 77, $3865-3868$.

(66) Giannozzi, P.; Baroni, S.; Bonini, N.; Calandra, M.; Car, R.; Cavazzoni, C.; Ceresoli, D.; Chiarotti, G. L.; Cococcioni, M.; Dabo, I.; Dal Corso, A.; de Gironcoli, S.; Fabris, S.; Fratesi, G.; Gebauer, R.; Gerstmann, U.; Gougoussis, C.; Kokalj, A.; Lazzeri, M.; Martin-Samos, L.; Marzari, N.; Mauri, F.; Mazzarello, R.; Paolini, S.; Pasquarello, A.; Paulatto, L.; Sbraccia, C.; Scandolo, S.; Sclauzero, G.; Seitsonen, A. P.;
Smogunov, A.; Umari, P.; Wentzcovitch, R. M. J. Phys.: Condens. Matter 2009, 21, 395502-395502.

(67) Anisimov, V. I.; Zaanen, J.; Andersen, O. K. Phys. Rev. B: Condens. Matter Mater. Phys. 1991, 44, 943-954.

(68) Walker, J. D. S.; Grosvenor, A. P. J. Solid State Chem. 2013, 197, $147-153$.

(69) Freer, R.; O’Reilly, W. Mineral. Mag. 1980, 43, 889-899.

(70) McClure, D. S. J. Phys. Chem. Solids 1957, 3, 311-317.

(71) Hartland, G. V. Chem. Rev. 2011, 111, 3858-3887.

(72) Della Valle, G.; Scotognella, F.; Kandada, A. R. S.; ZavelaniRossi, M.; Li, H.; Conforti, M.; Longhi, S.; Manna, L.; Lanzani, G.; Tassone, F. J. Phys. Chem. Lett. 2013, 4, 3337-3344.

(73) Scotognella, F.; Della Valle, G.; Srimath Kandada, A. R.; Dorfs, D.; Zavelani-Rossi, M.; Conforti, M.; Miszta, K.; Comin, A.; Korobchevskaya, K.; Lanzani, G.; Manna, L.; Tassone, F. Nano Lett. 2011, 11, 4711-4717.

(74) Ghosh, S. C.; Biesinger, M. C.; LaPierre, R. R.; Kruse, P. J. Appl. Phys. 2007, 101, 114322.

(75) Paolella, A.; Bertoni, G.; Dilena, E.; Marras, S.; Ansaldo, A.; Manna, L.; George, C. Nano Lett. 2014, 14, 1477-1483.

(76) Biesinger, M. C.; Payne, B. P.; Grosvenor, A. P.; Lau, L. W. M.; Gerson, A. R.; Smart, R. S. C. Appl. Surf. Sci. 2011, 257, 2717-2730.

(77) Yamashita, T.; Hayes, P. Appl. Surf. Sci. 2008, 254, 2441-2449. 